\title{
The beam emission spectroscopy diagnostic on the DIII-D tokamak
}

\author{
G. McKee, R. Ashley, R. Durst, R. Fonck, M. Jakubowski, and K. Tritz \\ University of Wisconsin-Madison, Madison, Wisconsin 53706 \\ K. Burrell, C. Greenfield, and J. Robinson \\ General Atomics, San Diego, California 92186-5608
}

(Presented on 10 June 1998)

\begin{abstract}
A beam emission spectroscopy system has been installed on DIII-D to provide localized density fluctuation measurements for long-wavelength turbulent modes with $k \leqslant 3 \mathrm{~cm}^{-1}$ which are typically associated with anomalous radial transport. High signal-to-noise fluctuations measurements are accomplished through use of high speed electronics to maintain a frequency response of over 500 $\mathrm{KHz}$ and cryogenically cooled amplifiers and detectors to reduce electronic noise. The optics and neutral beam-sightline geometry have been optimized to allow for spatial resolution of $\Delta r$ $\leqslant 1 \mathrm{~cm}$. In addition, a half-scale two-dimensional (2D) fiber array to measure the 2D turbulent density field, necessary to measure the full $S\left(k_{r}, k_{\theta}\right)$ wavenumber spectra, has been implemented and initial results obtained. (C) 1999 American Institute of Physics. [S0034-6748(99)65801-4]
\end{abstract}

\section{INTRODUCTION}

A new beam emission spectroscopy (BES) system has been installed at DIII-D and is now routinely operated in support of a wide variety of experiments. At present, the DIII-D BES system consists of 32 spatial channels and has been used to acquire data to study turbulence during internal transport barrier formation, $L$ to $H$-mode transitions, RI mode (radiative improved), dimensionless scaling studies, transport theory studies, as well as density fluctuation during magnetohydrodynamics (MHD) and disruption phenomena. The BES system was developed to measure spatially localized, long-wavelength density fluctuations in the core and edge regions of tokamak plasmas.

BES diagnostic systems have been previously deployed on several tokamaks: PBX-M, ${ }^{1}{ }^{\text {TFTR }},{ }^{2,3}$ Phaedrus-T, ${ }^{4}$ and TEXT-U. ${ }^{5}$ To deploy the BES diagnostic on DIII-D, numerous advances and technological innovations were required. The plasma rotation speeds encountered on DIII-D are typically much higher than those on other tokamaks with the high-power, all coinjection neutral beam system. High plasma densities are routine in the advanced confinement modes, thereby reducing beam penetration and BES light levels. The required advancements thus included higher frequency electronics to diagnose the more rapidly rotating plasmas, development of cryogenically cooled detectors to minimize electronic noise, optimized optics to increase spatial resolution, and new analysis techniques to extract low fluctuation signals in high-confinement plasmas. Taken together, these advances provide a new BES system on DIII-D whose capabilities are significantly advanced beyond those of previous BES systems on other experiments. In this article, the major subsystems of this improved BES apparatus are described. In addition, the ability to reconfigure the viewing optics to allow two-dimensional measurements of the density fluctuations is described and demonstrated with the limited spatial coverage presently available.

\section{DIII-D BES DIAGNOSTIC SYSTEM}

BES measures the local density by observing the collisionally excited emission from the deuterium beam particles as they traverse the plasma, interacting with plasma electrons and ions. The $D_{\alpha}$ transition $\left(n=3-2, \lambda_{0}=656.1 \mathrm{~nm}\right)$ of the beam particles is isolated from the intense edge $D_{\alpha}$ emission because of the doppler shifting of the emission to the blue by $0.5-3.5 \mathrm{~nm}$ due to the beam atom velocity and BES sightline viewing geometry. Fluctuations in the observed signal are directly proportional to the local density fluctuations through a proportionality constant that depends on local electron temperature and density: $d n / n=K\left(T_{e}, n_{e}\right) d I / I .^{6,7}$

The neutral beam viewing optical system consists of a custom-designed $f / 2, f=40 \mathrm{~cm}$, objective lens, $25 \mathrm{~cm}$ vacuum window, in-vessel shutter, optical axis-folding mirrors (to avoid a toroidal field coil) and scannable 2D motorized fiber-mounting array, all shown in Fig. 1. The fibermounting array places the fiber faces at the curved focal plane of the objective lens. The individual fiber bundles for each spatial channel can be deployed arbitrarily in the focal plane. The array can be remotely scanned radially to observe different spatial regions of the plasma on a shot-to-shot basis.

Four $1 \mathrm{~mm}$ fused-silica optical fibers in a square configuration collect light at $f / 2$ for a throughput of $0.6 \mathrm{~mm}^{2}$ ster spatial channel. The observed volume in the plasma is then defined by the intersection of the optical sightline with the neutral beam. The fiber images are focused at the center of the neutral beam and the roughly 4-to-1 magnification results in an observed spot size of about $1 \mathrm{~cm}^{2}$ in a poloidal cross section of the tokamak. The sightline is deployed so that the emission volume, which has a half-width of over $20 \mathrm{~cm}$ in the toroidal direction, is nearly tangent to a magnetic flux surface. Since turbulent eddies are expected to have long toroidal correlation lengths, no spatial resolution is lost. The $\sim 1 \mathrm{~cm}$ radial and vertical resolution allows for observation of long-wavelength modes with wavenumber $k \leqslant 3 \mathrm{~cm}^{-1}$. 


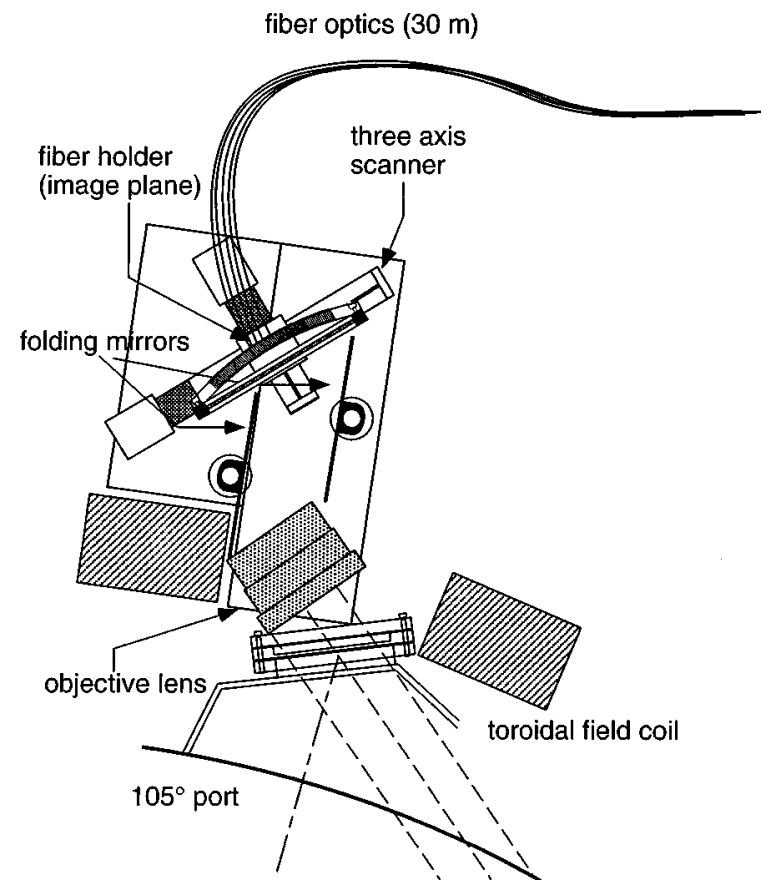

FIG. 1. Schematic of port optics showing objective lens, folding mirrors, remotely scannable fiber mounting array, and fiber optics to remotely located spectroscopy lab.

The sightlines are also angled at approximately $5^{\circ}$ in a vertical plane relative to the equatorial (horizontal) plane of the tokamak to more closely align the observation volume with the magnetic field lines. This optimizes the poloidal resolution for the usual magnetic geometry employed on DIII-D (plasma current opposite to toroidal field direction) for magnetic field pitch angles typically in the range of $5^{\circ}-10^{\circ}$. The 32 available spatial channels are typically deployed with 16 radial channels covering about $16 \mathrm{~cm}$, and two poloidal (vertical) arrays of 8 channels each, covering $8 \mathrm{~cm}$. DIII-D has a plasma radius of approximately $60 \mathrm{~cm}$ with the most interesting fluctuation data occurring in the outer $2 / 3$ of the plasma. Thus $2-3$ shots can fully spatially document fluctuations in a given plasma condition.

The approximately $40 \mathrm{~m}$ optical fibers transmit light to the remotely located spectrometers. The BES spectrometers are in a diagnostic laboratory adjacent to the DIII-D vessel that experiences an anisotropic neutron and gamma radiation flux during high-power plasma operation. Because the detectors are susceptible to radiation, a five-sided radiation shield with a four inch lead wall surrounded by two inches of borated polyethylene enclose the spectrometer/detector assemblies. The fibers are coupled to the spectrometers in this radiation shield.

Each spectrometer contains an $f / 2$ collimating lens, a 3 $\mathrm{nm}$ band pass $\lambda_{0}=654 \mathrm{~nm}$ interference filter, aspheric $f / 1$ focusing lens, and $\approx 1 \mathrm{~mm}^{2}$ photoconductive photodiode detector. The $3 \mathrm{~nm}$ bandpass filter is chosen to pass light from all three beam energy components and provide system-wide uniformity while rejecting the intense edge $D_{\alpha}$ radiation at $\lambda_{0}=656.1 \mathrm{~nm}$. The bremsstrahlung background is negligible compared to the beam emission, and there are no significant interfering spectral lines in this spectral band.
Each channel receives a light signal on the order of several nanowatts $\left(\approx 10^{10}\right.$ photons/s) with an output of nanoamp currents. Fast preamplifiers and signal conditioning circuits amplify this signal to of order $1 \mathrm{~V}$ for digitization while retaining a frequency response of over $0.5 \mathrm{MHz}$. The circuit design is similar to those utilized on the TFTR BES system, ${ }^{8}$ though they have been extended from a frequency response of up to $150 \mathrm{kHz}$ to approximately $750-800 \mathrm{KHz}$. The frequency response has been increased using lower impedance circuitry and faster components.

The signals are digitized with six 6-channel Joerger 3 $\mathrm{MHz}$ digitizers operating at $1 \mathrm{MHz}$, all synchronized to a common external clock and start trigger. Each channel has 512 Ksamples (12 bit) of memory allowing for a measurement time window of just over $0.5 \mathrm{~s}$ per shot. The preamplifier circuits can operate up to near $800 \mathrm{kHz}$, though active antialiasing filters presently cut off the signal at $450 \mathrm{kHz}$. Increasing this cutoff frequency would allow for higher frequency operation that may ultimately be required for core measurements in highly rotating DIII-D discharges, at the expense of the data acquisition time window. This may be required to make velocity fluctuation measurements utilizing wavelet analysis techniques being developed. ${ }^{9}$

The BES electronics require periodic calibration to measure the dark-noise spectrum and frequency response transfer function of the detector/amplifier circuits, as well as the fastchannel to slow-channel gain ratio. This is performed by illuminating the detector channels with a retractable bank of red light emitting diodes (LEDs) located in the spectrometer enclosure, driven by a dc signal with adjustable ac component.

To minimize preamplifier noise, the photodiode detector and first-stage preamplifier field effect transistor (FET) chip are cryogenically cooled to approximately $140 \mathrm{~K}$ during operation. The primary noise source is the first stage FET voltage noise ( $e$ noise) with both the voltage and current noise ( $i$ noise) being proportional to the square root of temperature. In addition, the $e$ noise is inversely proportional to the square root of the FET transconductance which increases with lower temperature as electron mobility increases. The photodiode dark current is also temperature dependent and is reduced at the lower temperatures. There is a lower operating temperature limit of near $100 \mathrm{~K}$ when silicon in the FET becomes an insulator as electrons no longer enter the conduction band, resulting in detector "freeze out." 10

Figure 2 shows the rms electronic noise level vs temperature demonstrating that noise power is reduced by a factor of about 3 through the cryogenfic cooling with most of the reduction occurring between 220 and $150 \mathrm{~K}$. A liquid nitrogen delivery system is connected to the detector assemblies to provide this cooling. The liquid nitrogen flow is controlled by thermostat to maintain the temperatures to within a $\pm 5 \mathrm{~K}$ degree range. To prevent condensation and freezing of ambient moisture, the detectors are housed under vacuum in an airtight box at a pressure of 50 mTorr or less. Since the spectrometers are in close proximity to the cryogenically cooled electronics, strip heaters are applied to the spectrometer enclosures to maintain them near room temperature so as 


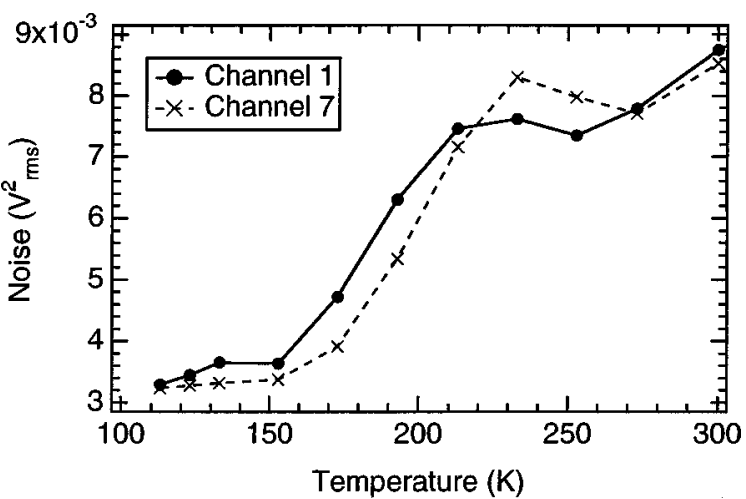

FIG. 2. Noise power as a function of temperature showing sharpest drop in noise in temperature range of $150-220 \mathrm{~K}$.

to prevent condensation on the lenses and changes in the spectral range of the interference filters.

\section{SMALL FLUCTUATION SIGNAL ISOLATION}

The high toroidal rotation on DIII-D, large electric fields, and resulting Doppler shifts tend to complicate fluctuation signal extraction methods previously developed on TFTR and other experiments. In the core plasma regions fluctuation levels are typically low $(\tilde{n} / n \leqslant 0.1 \%)$ and are Doppler-broadened spectrally. These effects are shown in the power spectra in Fig. 3. Figure 3(a) is from near the edge of a typical plasma with a well defined broadband fluctuation spectrum, while Fig. 3(b) is at the midradius. In cases where the spectra are spread out, as in Fig. 3(b), quantitative measurements require very accurate photon and electronic noise subtraction, while the higher frequency measurement of the photon noise level is no longer available. Small errors in either of these quantities can lead to large errors in the extracted fluctuation power.

A modified method of calculating cross-power spectra has been developed to extract fluctuation data from core measurements. Since the electronic and photon noise are uncorrelated between channels, taking the cross power largely eliminates these contributions to the signal. However, the cross-power between two random data sets with no shared signal at all still results in a finite, nonzero power. This offset from zero cross power arises from numerical bias errors in calculating the cross power, which is always a positivedefinite number. ${ }^{11}$ In cases were the photon and electronic noise are large compared to local fluctuation power, this residual cross-power signal can be significant or even dominate the actual, local plasma fluctuation power.

This nonzero bias error is measured from the data set and then subtracted by first performing a standard cross power, then time shifting one of the two input data sets by a time that is short compared to the evolution of the background plasma, but long compared to turbulence decorrelation times. Typically this is $0.5-2 \mathrm{~ms}$. The cross power of one channel and a "time-shifted" data set from the second channel then contains all of the residual cross power arising from numerical bias errors in the data, but none of the plasma fluctuation power since plasma turbulence correlation

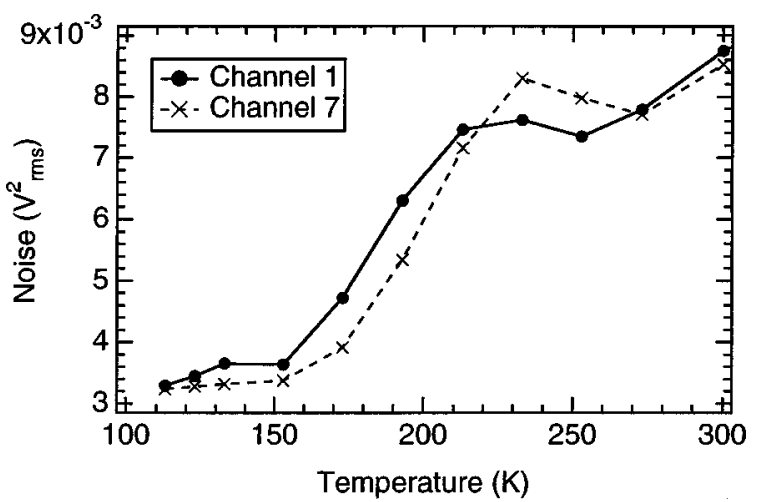

FIG. 3. (a) Typical turbulence spectrum at edge of plasma showing large fluctuations that are easily isolated from photon noise background, (b) spectrum from core channel $(\rho=0.65)$ showing broadening and reduction in relative intensity, (c) isolation of turbulent spectrum using noisecompensated crosspower method.

times are of the order 5-10 microsec. Subtracting these two cross powers then provides a very accurate measurement of the fluctuation power arising from plasma turbulence alone, even in the presence of large photon and electronic noise contributions to the signal.

The cross power spectra in Fig. 3(c) is the result of this background-subtracted spectrum for the channel shown in Fig. 3(b) and an adjacent channel, demonstrating the improved accuracy with which the plasma fluctuation power and amplitude can be derived relative to the autopower method. An uncorrected cross power would have included an unknown fraction of background contribution.

As first implemented, this method rests on the assumption that the two spatially adjacent channels are separated by a distance that is well less than the correlation length of the plasma, which is often reasonable to first order, but is still problematic for short correlation lengths. In the future, this limitation will be avoided by sending the light from a single spatial channel into two separate detectors. Standard crosscorrelation analysis of these two channels will allow for accurate autocorrelation measurements.

\section{2D MEASUREMENTS OF TURBULENCE}

Turbulence in a toroidally confined tokamak plasma is fundamentally $2 \mathrm{D}$ in nature. Furthermore, it is desirable to acquire full 2D measurements to obtain a true $S\left(k_{r}, k_{\theta}\right)$ spectrum for comparison to theory, provide time-averaged and possibly time-resolved visualization of the turbulent eddy structures, obtain high spatial resolution fluctuation velocity and velocity shear measurements, and ultimately characterize the turbulence growth and nonlinear energy transfer rates for more direct comparison to nonlinear theory.

Most measurements of turbulence in the core region of hot tokamak plasmas are one dimensional in nature; they measure the wave number spectrum and/or correlation properties in either the radial or poloidal directions separately. $\mathrm{BES}$ is one of the few diagnostic techniques which provides access to both directions for turbulence studies, but previously, the closest approach to realizing 2D operation has been the use of crossed single radial and poloidal arrays. In 


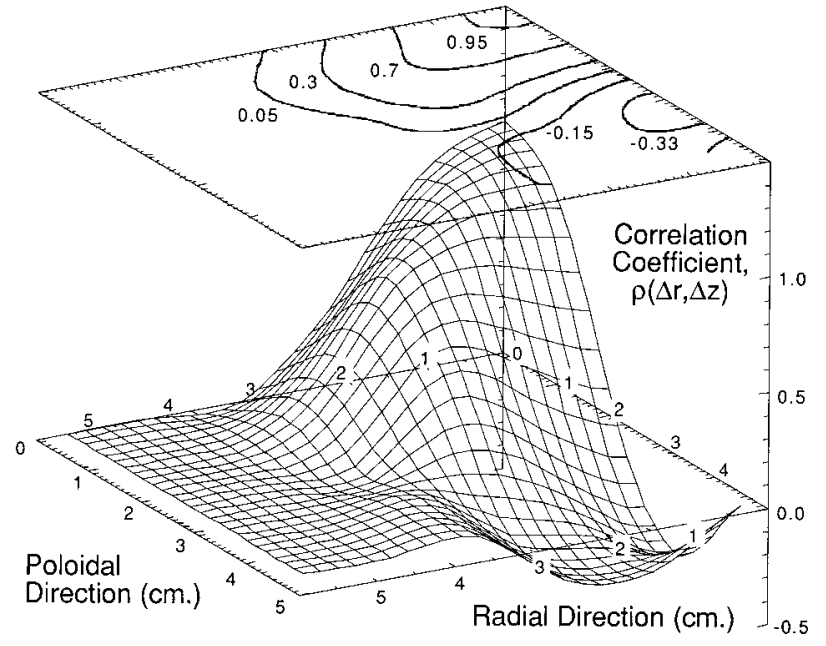

FIG. 4. Initial measurement of the $2 \mathrm{D}$ correlation function, $\rho(\Delta r, \Delta z)$ near the edge of an $L$-mode plasma at $\rho=0.9-1.0$ showing wavelike poloidal spectrum and decaying radial spectrum, with data splined and smoothed to improve visualization.

this case, all information of the phase between the radial and poloidal modes has been lost by integration along perpendicular directions.

The DIII-D BES system has been reconfigured to provide a very low-resolution $2 \mathrm{D}$ turbulence measurement. While limited to the present total of 32 sample points, this does allow for the first time direct measurements of 2D structures in turbulence in large tokamaks and demonstrates the promise of further development in this direction. Spatial channels are arranged so as to provide the nominal spatial resolution of $\Delta r \approx 1 \mathrm{~cm}$. The 2D fiber mounting array is located in the fiber holder discussed earlier and has the proper radius of curvature and angle to the face to match the focal field of the objective lens and optimize optical throughput. The array size is 6 spatial channels radially and up to 15 poloidally. The existing $32 \mathrm{BES}$ spatial channels can be deployed in, for example, a $5 \times 6$ or an $8 \times 4$ array. The configuration can be readily changed from one experiment to the next.

Initial 2D measurements have been obtained in an $L$ mode plasma, and the resulting $2 \mathrm{D}$ correlation function evaluated. This function, $\rho(\Delta r, \Delta z)$, is shown in Fig. 4. Each point is evaluated by calculating the correlation function between a reference channel at the corner of the array and a given channel at zero time delay. These measurements were obtained with a 6 (radial) $\times 5$ (poloidal) array, covering a roughly $6 \times 5 \mathrm{~cm}$ region near $\rho=0.9-1.0$, just inside of the separatrix near the outer plasma midplane. The resulting data have been smoothed and splined to a finer spatial resolution grid for optimal visualization. This 2D correlation function shows an oscillatory poloidal and purely decaying radial structure, as might be expected from previous onedimensional (1D) measurements. ${ }^{12}$ To fully map the poloidal structure, it can be seen that greater poloidal coverage will be necessary since the correlation function remains nonzero over the $5 \mathrm{~cm}$ covered. The analysis routines to obtain the full $S\left(k_{r}, k_{\theta}\right)$ function by deconvolving the spatial sampling volume are being developed.

\section{ACKNOWLEDGMENTS}

We appreciate the support of the entire DIII-D program for supporting this collaboration through experimental run time and on-site hardware and software support. This work was supported by U.S. D.O.E. Grant No. DE-FG0289ER53296 and Contract No. DE-AC03-76-89ER51114.

${ }^{1}$ R. J. Fonck, P. A. Dupperex, and S. F. Paul, Rev. Sci. Instrum. 61, 3487 (1990).

${ }^{2}$ R. D. Durst, R. J. Fonck, G. Cosby, H. Evensen, and S. F. Paul, Rev. Sci. Instrum. 63, 4907 (1992).

${ }^{3}$ S. F. Paul and R. J. Fonck, Rev. Sci. Instrum. 61, 3496 (1990).

${ }^{4}$ H. Evensen, D. Brouchous, D. Diebold, M. Doczy, R. J. Fonck, and D. Nolan, Rev. Sci. Instrum. 63, 4928 (1992).

${ }^{5}$ R. D. Durst, E. A. Denhartog, R. J. Fonck, J. S. Kim, and Y. Karzhavin, Rev. Sci. Instrum. 66, 842 (1995).

${ }^{6}$ R. K. Janev, C. D. Boley, and D. E. Post, Nucl. Fusion 29, 2125 (1989).

${ }^{7}$ T. A. Gianakon, R. J. Fonck, J. D. Callen, R. D. Durst, and J. S. Kim, Rev. Sci. Instrum. 63, 4931 (1992).

${ }^{8}$ R. J. Fonck, R. Ashley, R. Durst, S. F. Paul, and G. Renda, Rev. Sci. Instrum. 63, 4924 (1992).

${ }^{9}$ M. Jakubowski, R. Fonck, J. S. Kim, and G. McKee, Rev. Sci. Instrum. (these proceedings).

${ }^{10}$ A. T. Lee, Rev. Sci. Instrum. 60, 3315 (1989).

${ }^{11} \mathrm{~J}$. Bendat and A. Piersol, Engineering Applications of Correlation and Spectral Analysis (Wiley, New York, 1993).

${ }^{12}$ R. J. Fonck et al., Phys. Rev. Lett. 70, 3736 (1993). 